\title{
A review on nanotechnology and its application in modern veterinary science
}

\author{
Kalkidan Mamo Woldeamanuel, Fufa Abunna Kurra and \\ Yonas Tolosa Roba*
}

College of Veterinary Medicine and Agriculture, Addis Ababa University, P.O. Box 34, Bishoftu, Ethiopia

Received: 06 February, 2021
Accepted: 24 February, 2021
Published: 25 February, 2021

*Corresponding author: Yonas Tolosa Roba, College of Veterinary Medicine and Agriculture, Addis Ababa University, P.O. Box 34, Bishoftu, Ethiopia, Tel: +251944264287; E-mail: yonas.tolosa@aau.edu.et

Keywords: Animal-health; Nanoparticles; Nanotechnology; Veterinary

https://www.peertechzpublications.com

\section{Check for updates}

\section{Abstract}

The term nanotechnology refers to material processing on the atomic or molecular scale, especially for the construction of microscopic level devices with the ability to calculate, function, and organize. The microscopic level typically refers to the size range of 1-100 nm. We opt to review the application of nanotechnology in veterinary medicine for this specific article and highlight their functions in animal health and production improvement. Nanotechnology plays a key role in veterinary medicine and other areas of animal production and also plays a significant role in the treatment of diseases through the development of a system for the delivery of smart medicines. Nanotechnology has revolutionized virtually all veterinary medicine and animal science disciplines these days by offering new, small-scale devices and materials that are beneficial to living organisms. Nanoparticles used for disease detection, treatment, delivery of medications, animal breeding, and reproduction include quantum dots, magnetic nanoparticles, nanopores, polymeric nanoparticles, nanoshells, fullerenes, liposomes, and dendrimers. While nanotechnology is regarded as one of the foremost technologies already applied in diverse subjects, its application in veterinary science is still in its infancy stages when compared to other sister disciplines.

\section{Introduction}

The term nanotechnology refers to the nanoscale-level ability to calculate, function, and organize the matter. The scale usually refers to the matter in the size range of $1-100 \mathrm{~nm}$ in at least one dimension but is often extended to include materials in size below $1 \mathrm{~nm}[1,2]$. It is not limited to a specific sector; rather, it is an enabling collection of technologies, which cross all sectors of activity and scientific disciplines. Nanotechnology uses the philosophy and techniques of the nanoscale to understand and transform biosystems, which use biological concepts and materials to build new nanoscale devices and systems [3,4].

Rationally engineered nanostructures are among the most impressive man-made materials and unveil distinctive chemical, physical, and/or biological characteristics [5]. Such characteristics make it possible to use the nanostructures for an exceptional number of applications in various sectors including electronics, agriculture, and health care [6].

One of the key benefits of nanotechnology is to close the gap between the worlds of macroscopy and microscopy, where nanoparticles are the perfect medium to communicate with biological systems [7]. Nanoparticles have different properties that differentiate them from bulk materials that include large active surfaces, easily controllable surface chemistry that allows binding to small molecular drugs, imaging labels, and ligands such as antibodies, peptides, nucleic acids. Also, their small size allows for exclusive intracellular and extracellular interactions, such as extravasation via endothelial cells and increased permeability and retention in tumor tissues [8-10].

It is believed that nanotechnology will deliver a large number of breakthroughs in the twenty-first century that will advance the habit of clinical veterinary medicine and have the ability to modernize veterinary medicine, animal welfare and other areas of animal production [11]. Veterinary nanotechnology will enhance the systems of diagnosis and treatment delivery, provide new gears for molecular and cellular breeding, animal history from birth to consumer table, animal nutrition scenarios ranging from nutrient uptake, and use, animal waste adjustment as expelled from livestock, pathogen detection, and much more [12]. In this paper, we opt to review the application of nanotechnology in veterinary medicine and highlight their roles on animal health and production improvement. 


\section{Classifications of nanoparticles}

Nanoparticles are scale-sized microscopic particles of $1 \mathrm{~nm}$ to $100 \mathrm{~nm}[1,2,13]$. Particles such as nanocrystals, polymers, dendrimers, silica oxides, carbon, metal oxides, lipids, and quantum dots have been developed over the last few decades on based on various components, along with an increasing variety of newly developed materials [14]. Some of the widely used nanoparticles were addressed below.

\section{Fullerenes}

Fullerenes are nanoparticles that consist entirely of molecules formed from carbon. Their nanomedicine capabilities are extensively studied, and their use in the manufacturing sector has already been established [15]. Carbon nanoparticles combine a broad variety of characteristics that can be useful in therapy, and regenerative medicine, including high aspect ratio, thermal, conductive and mechanical properties. One of the most commonly used nanotechnology fullerenes is Carbon Nanotubes (CNTs), which may be single or multi-walled (SW, MW). SWCNTs provide a new approach to the delivery of drugs because of their ability to reach cells in a 'needle-like' manner [16] and by diminishing their length they can gain nuclear exposure. This phenomenon is extremely useful since CNTs can be conjugated or charged with drugs to improve therapeutic effectiveness $[16,17]$.

\section{Quantum dots}

Quantum dots are nanocrystals that measure between 2-10nm and can fluorescence when excited by light [18]. Their composition consists of an inorganic center, whose size defines the color of an inorganic shell emitted and an aqueous organic coating to which biomolecules are combined. It can be used as a diagnostic as well as a clinical method for biomedical purposes and can also be used for the imaging of sentinel nodes in patients with cancer to stage tumors and to scheme therapy [19].

\section{Liposomes}

Liposomes are synthetic vesicles of nanoscale size and a spherical form consisting of natural phospholipids and cholesterol [20]. Liposomes were the first to be tested as carriers of the drugs. They are micro-particular or colloidal carriers, typically with a size range of $80-300 n m$ [21]. These can be used as effective systems for the administration of drugs. Cancer chemotherapeutic and other toxic medicines, such as amphotericin and hamycin, show greater efficacy and protection when used as liposomal medicines compared to traditional preparations [22].

\section{Magnetic nanoparticles}

Magnetic nanoparticles, such as iron oxide paramagnetic compounds are promising candidates for disease treatment due to the ability of antibodies to bind to their surface and the possibility of targeting using an external magnetic field [13]. The most capable substances tend to be superparamagnetic iron oxide nanoparticles of less than $10 \mathrm{~nm}$ in diameter and with superior magnetic properties. They are small, thermally agitatedw magnets called "ferromagnetic fluids" or "ferrofluids" in liquids. Superparamagnetism only occurs through the presence of a magnetic field; if this is removed, the magnetization will vanish, particles will cease to interact, and thus potential vascular embolization can be avoided [13,23].

\section{Nanopores}

Nanopores were conceived by Desai and Ferrari in 1997 [24]. Consists of high porous density wafers reaching up to 20nm in diameter. The pores allow for the flow of oxygen, glucose, and other items, such as insulin. This does not require immunoglobulins and cells to move through them, though. Nanopores can be used as a tool to protect grafted tissues from the host defense system. $\beta$ pancreatic cells may be enfolded inside the nanopore system and inserted in the body of the recipient. This tissue sample absorbs the nutrients from the nearby tissues while remaining invisible by the defense system and hence escapes from rejection. It can serve as a newer treatment tool for insulin-dependent diabetes mellitus [25].

\section{Nanoshells}

Nanoshells had been produced in West and Halas [26]. Nanoshells consist of silica nucleus nanoparticles, and a thin metal layer covering. This can be applied to appropriate tissue using immunological methods. This technique is under analysis for tumor therapy. Used nanoshells that are designed to absorb infrared rays when bared from a source outside the body to exhibit the nanoshell's Thermo ablative property [27].

\section{Applications of nanotechnology in veterinary science}

Nanotechnology is used for the production of nanoscale drugs, controlled delivery systems, contaminant detection, and for the design of molecular and cellular biology nanodevices [28]. It will have a major role in animal welfare, veterinary medicine, and other animal production fields, and will also play an essential role in disease control through the implementation of a smart drug delivery system. One practical function of nanotechnology in medicine, which is currently being developed, involves the use of nanoparticles to administer drugs or other constituents to specific cell types. Particles are programmed to be drawn to diseased cells that cause certain cells to be handled directly [29]. Some of the veterinary science's major nanotechnological approaches have been reviewed below

\section{Nanovaccines}

The nanovaccine is emerging as a new solution to the vaccination technique. Nanovaccines can stimulate both humoral and cell-mediated immune response and are more effective than traditional vaccines. They have the promise of channeling the body's immune system to combat pathogens and avoid the spread of infections and diseases [30,31]. The current method of vaccination has changed from live and killed organisms practice to a much safer candidate for synthetics and recombinants. Such new candidates for vaccine alone are often poorly immunogenic and vulnerable to degradation and need an engineered adjuvant that enhances immunogenicity [32]. 
Due to the un-adjustability of conventional adjuvants, the introduction of nanotechnology has brought about a series of novel antigen-carrying strategies. Such adjuvants based on nanoparticles can be designed for reduced dose frequency and a comfortable route of administration to induce a particular target immune response, for instance, the intranasal route to improve target mucosal immunity. This makes them particularly suitable for veterinary medicine where large numbers of animals will need to be handled at once, or where vaccination by traditional means is impractical due to comprehensive management systems or lack of accessibility [33].

\section{Nanopharmacuetics}

Pharmacology and nanophamaceuticals are at the forefront of what nanotechnology can develop in comparison to the other veterinary medicine field [34]. Considering the field of pharmacology, it is important to reiterate that nanotechnology enables the production of new drugs and the possibility of reworking traditional substances to achieve better results in efficacy [35]. The pharmacokinetics and therapeutic index of the medicines can be greatly enhanced by inserting medications onto nanoparticles by physical encapsulation, adsorption, or chemical conjugation, as opposed to the free product equivalents. Drug-charged nanoparticles that invade host cells by endocytosis and then release drug payloads to treat intracellular infections that are caused by microbes [36].

Drug delivery based on nanoparticles provides many benefits, such as enhancing drug-therapeutic efficacy and pharmacological properties. Several nano-drug delivery systems have shown the effectiveness of nanoparticles in enhancing pharmacokinetics, reducing unwanted side effects, and optimizing transmission to disease sites [37]. The required dosage of the drug is used in this procedure and side effects are greatly minimized, as the active agent is concentrated only in the morbid area. This highly targeted approach will reduce patients' costs and pain [38].

In turn, the use of nanoparticulate drug carriers will overcome many important drug delivery problems including enhancing drug solubility and safety, increasing the half-lives of medications in the blood, reducing adverse effects in nontarget organs, and focusing medications at the location of the disease [39]. Drugs may be spread in a gel, encapsulated in a vesicle, dissolved in a hydrophobic nucleus, or bound to a nanoparticle sheet. Many drug delivery mechanisms based on nanoparticles, including liposomes, polymeric nanoparticles, dendrimers, ceramic-containing capsules, micelles, and others have been used to transport therapeutic agents for small molecules, peptides, and oligonucleotides [40].

Liposomes were the first to be investigated as carriers of the drugs, which are spherical vesicles consisting of phospholipids and steroid, bilayers, or certain surfactants which shape spontaneously as other lipids are distributed in aqueous media where liposomes may be formed [41]. Liposomes have been reported to enhance drug solubility and boost their pharmacokinetic properties, such as the therapeutic index of chemotherapeutic drugs, accelerated synthesis and reduction of adverse side effects, and an improvement in in vitro and in vivo anticancer behavior. The encapsulation process embeds a drug in liposomes. The release of a drug from liposomes depends on the structure of the liposome, the $\mathrm{pH}$, the osmotic gradient, and the atmosphere surrounding it [42]. Liposomal associations with cells can be understood through adsorption, fusion, endocytosis, and transition of lipids. Liposomal forms, such as anticancer medications, neurotransmitters, antibiotics, and anti-inflammatory medicines, have several medication sources [43].

Molecule variety retains possible therapeutic interest from which dendrimers grab wide applicability in drug conveyance. Dendrimers possess several versatile divisions that contain voids where the drug molecules can be stuck physically [44] This compact construction makes for fantastic encapsulation. In nanotechnology, their structure made a major impact in providing well-controlled practical building blocks. Through drug distribution and cancer treatment, they have a variety of uses. Dendrimers are successfully used in drug distribution as they convey a drug at a precise rate by chemical modulation either by adjustment of hydrolytic release conditions and selective outflow of drug molecules depending on their size or form [45]. High-load dendrimers have demonstrated rapid pharmacological reactions with enhanced effectiveness [46].

Equivalently, carbon nanotubes with better strength and steadiness can also serve as product carriers. Binding antibodies to carbon nanotubes with fluorescent or radiolabeling will achieve in cell specificities [47]. Nanotubes joining the cell may be directed by endocytosis or by cell membrane penetration. By integrating carboxylic or ammonium groups into their arrangement, carbon nanotubes can be made more soluble and can be used for the transport of proteins and other drug molecules. Indium-111 carbon nanotubes labeled radionuclides are being investigated for selective killing of cancer cells [48].

\section{Animal breeding and reproduction}

Nanotechnology has started to flourish in the reproductive and reproduction sectors [49]. The goals of such nanotechnology-based animal reproduction investigations are to characterize nanoscale features of gamete cells using atomic force microscopy and similar scanning microscopy techniques, build nano-bio sensors for physiological or altered detection of the reproductive status [50], develop chemical tactics for the production of metal nanoparticles for fertility control applications, develop nanodevices for secure cryopreservation of gametes and embryos [51] and develop sustained release systems of molecules, including hormones, vitamins, antibiotics, antioxidants, nucleic acids, among others [52].

Breeding management is a costly and time-consuming problem for dairy farmers and pig farmers. One approach being tested now is a nanotube inserted under the skin to provide real-time monitoring of changes in blood estradiol levels. The nanotubes are used as a means of tracking estrus in animals, as these tubes are capable of binding and detecting the estradiol antibody by near-infrared fluorescence at the time of estrus.

Citation: Woldeamanuel KM, Kurra FA, Roba YT (2021) A review on nanotechnology and its application in modern veterinary science. Int J Nanomater Nanotechnol 
This sensor's signal will be integrated as part of a central breeding monitoring and control system for actuation [53]. In addition, nanotechnology devices such as microfluidics, nanoparticles, bioanalytic nano-sensors, can to solve even more puzzles related to animal health, growth, reproduction, and disease prevention and treatment [54]. Microfluidic and nanofluidic [55] are modern methods to improve conventional in vitro fertilization procedures and the development of in vitro embryos [56]. Recent reports have shown the usefulness of microfluidics in insulating motile sperm without centrifugation [57].

\section{Disease diagnostics}

In veterinary medicine, diagnosing a disease may take days, weeks, or even months as in the case of chronic diseases without any clinical symptoms. Hence, an infection may have grown by that time with the need to kill the entire herd Nanotechnology operates on the same scale as a virus or disease-infecting particle and therefore has the potential to be detected and eradicate very early. Hence, nanotechnology, for sensitive clinical diagnosis can be a successful tool [58]. In the one health thought, the use of nanotechnology instruments for the examination of animal diseases or as animal simulations for the diagnosis of human diseases is remarkable. Latest studies propose the use of quantum dots in small animal models for in vivo imaging [4].

Single-Photon Emission Computed Tomography (SPECT) and Positron Emission Tomography (PET) are nuclear medicine imaging techniques, which provide metabolic and functional information unlike the Computed Tomography (CT) and Magnetic Resonance Imaging (MRI), which provide only anatomical information. However, the combining of SPECT and PET with CT and MRI provides both detailed anatomical and metabolic information $[7,8]$.

To control disease progression before it becomes apparent with conventional morphological imaging techniques or laboratory tests, it is necessary to perform functional molecular alterations in vivo by using non-invasive, specific molecular imaging modalities providing anatomical and physiological in vivo information [59]. Nuclear medicine offers these molecular imaging methods by observing the body delivery of radiopharmaceutical compounds (gamma and positronemitters) delivered to the patient and can be visualized by SPECT or PET scanners $[60,61]$.

\section{Cancer treatment and diagnosis}

Cancer is a common illness and has been researched extensively. Traditional chemotherapeutic agent treatments have an impact on patients with many toxicity issues because they are not selective to tumor cells. The goal is to find a way to overcome the problem while building a method that can kill cancer cells with therapeutics whereas preventing healthy ones. Nanotechnology has been described as a modern, intelligent technology that creates devices with the potential to deliver drugs to different body locations. Such systems include submicron nanoparticles made up of multiple materials or devices [62].
Nanoparticles have a special property of a high surfaceto-volume ratio, enabling different functional groups to attach themselves to a nanoparticle and thus bind to certain tumor cells. The 10 to $100 \mathrm{~nm}$ small size of nanoparticles, in turn, helps them to be gathered preferentially at tumor sites because tumors lack an adequate lymphatic drainage network. Multifunctional nanoparticles that can diagnose, envision, and then treat a tumor in potential cancer therapy can be produced [63].

Imaging cancer is important for directing treatment plans and measuring the efficacy of the treatments being prescribed. The use of nanoparticles for comparing and enhancing images has allowed traditional modalities such as MRI and ultrasound to enhance cancer imaging and has also created new techniques such as optical-based cancer detection imaging [64]. The treatment's efficacy is specifically tied to the capacity of the medication to attack and destroy cancer cells while keeping healthy cells intact. Consequently, the high degree of cancer cell selectivity would be one of the most significant characteristics of novel anticancer agents. In this field, the convergence of nanotechnology with medicine represents a promising path to improving cancer therapy [65].

\section{Conclusion}

To conclude, nanotechnology is a fascinating and quickly evolving aspect of engineering that enables us to interact at the radioactive, and molecular levels to explore, manage, and apply nanometer-dimensional. It has opened up new prospective applications in biotechnology and molecular biology. Nanotechnology has revolutionized nearly all of the veterinary and animal science disciplines specifically in the developed countries by providing in-depth information and showing what is going on inside the deeper body of an organism. Nanoparticles used for disease diagnosis, treatment, delivery of drugs, animal breeding, and reproduction include quantum dots, magnetic nanoparticles, nanopores, polymeric nanoparticles, nanoshells, fullerenes, liposomes, and dendrimers. While nanotechnology is considered one of the big advances now applied in various fields, relative to other sister disciplines, it is still in the early stages of its application to veterinary science. Furthermore, the complexity of the technology to use and its high cost rendered particularly the developing countries to apply the technology in their anima science sector.

\section{References}

1. Jeevanandam J, Barhoum A, Chan YS, Dufresne A, Danquah, Beilstein MK (2018) Review on nanoparticles and nanostructured materials: history, sources, toxicity, and regulations. J Nanotechnol 9: 1050-1074. Link: https://bit.ly/37Gf13y

2. Boulaiz H, Alvarez P, Ramirez A, Marchal J, Prados J, et al. (2011) Nanomedicine: Application areas and development prospects. Int $\mathrm{J}$ Mol Sc 12: 3303-3321. Link: https://bit.ly/3dH5mxN

3. Chauhan R, Sharma G, Rana J (2010) Nanotechnology in health and disease. Bytes and Bytes Bareilly UP India 1-11.

4. Bentolila L, Ebenstein Y, Weiss S (2009) Quantum dots for in vivo small-anima imaging. J Nucl Med 50: 493-496. Link: https://bit.ly/3dGUAY9

Citation: Woldeamanuel KM, Kurra FA, Roba YT (2021) A review on nanotechnology and its application in modern veterinary science. Int J Nanomater Nanotechnol 
5. Albanese A, Tang P, Chan C (2012) The effect of nanoparticle size, shape, and surface chemistry on biological systems. Annu Rev Biomed Eng 14: 1-16. Link: https://bit.ly/37L2USS

6. Gupta N, Fischer R, George S, Frewer L (2013) Expert views on social responses to different applications of nanotechnology. J Nanopart Res 15: 1838. Link: https://bit.ly/3dKSK8G

7. Shreya G, Christopher G, Feng C, Weibo C (2016) Positron emission tomography and nanotechnology: A dynamic duo for cancer theranostics. Adv Drug Deliv Rev 3: 20. Link: https://bit.ly/2NQoVIZ

8. Cai W, Chen X (2007) Nanoplatforms for targeted molecular imaging in living subjects. Small 3: 1840-1854. Link: https://bit.ly/3ussG8|

9. Chakravarty R, Goel S, Dash A, Cai W (2017) Radiolabeled inorganic nanoparticles for positron emission tomography imaging of cancer: an overview. Q J Nucl Med Mol Imaging 61: 181-204. Link: https://bit.ly/3aOjRh8

10. Goel S, England CG, Chen F, Cai W (2017) Positron emission tomography and nanotechnology: A dynamic duo for cancer theranostics. Advanced drug delivery reviews 113: 157-176. Link: https://bit.ly/3qQnbhy

11. Wilczewska AZ, Niemirowicz K, Markiewicz KH, Car H (2012) Nanoparticles as drug delivery systems. Pharmacol Rep 64: 1020-1037. Link: https://bit.ly/3aQ7cue

12. Scott NR (2007) Nanoscience in veterinary medicine. Vet Res Commun 31 139-144. Link: https://bit.ly/3dPtvSy

13. Mahmoudi $M$, Hosseinkhani $H$, Hosseinkhani $M$, Boutry $S$, Simchi $A$, et al. (2011) Magnetic resonance imaging tracking of stem cells in vivo using iron oxide nanoparticles as a tool for the advancement of clinical regenerative medicine. Chem Rev 111: 253-280. Link: https://bit.ly/2NYw21T

14. Baptista PV (2009) Cancer nanotechnology-prospects for cancer diagnostics and therapy. Curr Canc Ther Rev 5: 80-88. Link: https://bit.ly/3khMWEQ

15. Madani S, Naderi N, Dissanayake O, Tan A, Seifalian A (2011) A new era of cancer treatment: Carbon nanotubes as drug delivery tools. Int J Nanomed 6 : 2963-2979. Link: https://bit.ly/3uDetWi

16. Beg S, Rizwan M, Sheikh A, Hasnain M, Anwer K, et al. (2011) Advancement in carbon nanotubes: Basics, biomedical applications, and toxicity. J Pharm Pharmacol 63: 141-163. Link: https://bit.ly/3uvCXAO

17. Madani S, Tan A, Dwek M, Seifalian A (2012) Functionalization of singlewalled carbon nanotubes and their binding to cancer cells. Int J Nanomedicine 7: 905-914. Link: https://bit.ly/37lkPd2

18. Iga AM, Robertson JH, Winslet MC, Seifalian AM (2007) Clinical potential of quantum dots. J Biomed Biotechnol 2007: 7087. Link: https://bit.ly/37HV9gO

19. Gao X, Cui R, Levenson W, Chung NS (2004) Invivo cancer targeting and imaging with semiconductor quantum dots. Nat Biotechnol 22: 969-976. Link https://bit.ly/3aQFA8u

20. Akbarzadeh A, Rezaei-Sadabady R, Davaran S, Joo S, Zarghami N, et al. (2013) Liposome classification, preparation, and applications. Nanoscale Res Lett 8 : 102. Link: https://bit.ly/3urUKIO

21. Sunderland C, Steiert M, Talmadge J, Derfus A, Barry S (2006) Targeted nanoparticles for detecting and treating cancer. Drug Dev Res 67: 70-93. Link: https://bit.ly/3pQ9jIS

22. McCormack B, Gregoriadis G (1994) Drugs-in-cyclodextrinsin liposome a nove concept in drug-delivery. Int J pharm112: 49-58. Link: https://bit.ly/2ZKWKhl

23. Mahmoudi M, Simchi A, Imani M, Milani A, Stroeve P (2008) Optimal design and characterization of superparamagnetic iron oxide nanoparticles coated with polyvinyl alcohol for targeted delivery and imaging. J Physic Chem B 112 14470-14481. Link: https://bit.ly/37GPnMa
24. Desai T, Chu W, Tu J, Beattie G, Hayek A, et al. (1998) Microfabricated immunoisolating biocapsules. Biotechnol Bioeng 57: 118-120. Link: https://bit.ly/3bwxAbS

25. Leoni L, Desai T (2001) Nanoporous biocapsules for the encapsulation of insulinoma cells: Biotransport and biocompatibility considerations. Trans Biomed Eng 48: 1335-1341. Link: https://bit.ly/3qRLxY2

26. West JL, Halas NJ (2000) Applications of nanotechnology to biotechnology commentary. Curr Opin Biotechnol 11: 215-217. Link: https://bit.ly/3kgR20d

27. Hirsch L, Stafford R, Bankson J, Sershen S, Rivera B, et al. (2003) Nanoshellmediated near-infrared thermal therapy of tumors under magnetic resonance guidance. Proc Natl Acad Sci USA 100: 13549-13554. Link: https://bit.ly/2ZldJkq

28. Assadi P (2008) A novel multiplication algorithm in nanotechnology. J Appl Sci 8: 2625-2630. Link: https://bit.ly/3soc4wu

29. Sahoo S, Ma W, Labhasetwar V (2004) Efficacy of transferrin-conjugated paclitaxel loaded nanoparticles in a murine model of prostate cancer. Int $\mathrm{J}$ Cancer 112: 335-340. Link: https://bit.ly/3aQ8F3।

30. Sekhon B, Saluja V, Sekhon BS (2011) Nanovaccines- An overview. Int J Pharm Frontier Res 1: 101-109. Link: https://bit.ly/3socjYq

31. Gheibi Hayat SM, Darroudi M (2019) Nanovaccine: a novel approach in immunization. J Cell Physiol 234: 12530-12536. Link: https://bit.ly/2P8L7Ph

32. Nordly P, Madsen H, Nielsen H, Foged C (2009) Status and future prospects of lipid-based particulate delivery systems as vaccine adjuvants and their combination with immunostimulators. Expert Opin on Drug Deliv 6: 657-672. Link: https://bit.ly/2P71hZs

33. Underwood C, Van E (2012) Nanomedicine and veterinary science: The reality and the practicality. Vet J 193: 12-23. Link: https://bit.ly/2ZM35cw

34. Feneque J (2000) Nanotechnology: A new challenge for veterinary medicine Pet Tribune 6: 16

35. Chen K, Conti P (2010) Target-specific delivery of peptide-based probes for PET imaging. Adv Drug Deliv Rev 62: 1005- 1022. Link: https://bit.ly/2NTKWqv

36. Zhang L, Pornpattananangkul D, Hu C, Huang C (2010) Development of nanoparticles for antimicrobial drug delivery. Curr Med Chem 17: 585-594. Link: https://bit.ly/3qTZ81k

37. McMillan J, Batrakova E, Gendelman H (2011) Cell delivery of therapeutic nanoparticles. Prog Mol Biol Transl Sci 104: 563-601. Link: https://bit.ly/3sszXmR

38. Cavalcanti A, Shirinzadeh B, Freitas R, Hogg T (2008) Nano robo architecture for medical target identification. Nanotechnology 19: 15. Link: https://bit.ly/3uB3AnZ

39. Muller RH, Keck CM (2004) Challenges and solutions for the delivery of biotech drugs-A review of drug nanocrystal technology and lipid nanoparticles. J Biotechnol 113: 151-170. Link: https://bit.ly/3byHfi7

40. Sahoo S, Labhasetwar V (2003) Nanotech approaches to delivery and imaging drug. Drug Discov Today 8: 1112-1120. Link: https://bit.ly/2NTAqPR

41. Silva R, Ferreira H, Cavaco-Paulo A (2011) Sonoproduction of liposomes and protein particles as templates for delivery purposes. Biomacromolecules 12 : 3353-3368. Link: https://bit.ly/37GhQSc

42. dos Santos GC, de Oliveira RE, Ribeiro RT, Leite EA, Lacerda RG, et al (2011) Study of the pilot production process of long-circulating and $\mathrm{pH}$ sensitive liposomes containing cisplatin. J Liposome Res 21: 60-69. Link: https://bit.ly/3aL7cvv

43. Yukihara M, Ito K, Tanoue O, Goto K, Matsushita T, et al. (2011) Effective drug delivery system for Duchene muscular dystrophy using hybrid liposomes

Citation: Woldeamanuel KM, Kurra FA, Roba YT (2021) A review on nanotechnology and its application in modern veterinary science. Int J Nanomater Nanotechnol Nanomed 7(1): 026-031. DOI: https://dx.doi.org/10.17352/2455-3492.000041 
including gentamicin along with reduced toxicity. Biol Pharm Bull 34: 712-716. Link: https://bit.ly/3dH8o59

44. Bianco A, Kostarelos K, Prato (2005) Applications of carbon nanotubes in drug delivery. M Biopolymers 9: 674 .

45. Amazi H, Addi (2005) Dendrimers of citric acid and poly (ethylene glycol) as the new drug-delivery agents. Biomaterials 26: 1175. Link: https://bit.ly/3dlktqM

46. Dilbaghi N, Kaur H, Kumar R, Arora P, Kumar S (2013) Nanoscale device for veterinary technology: Trends and future prospective. Adv Mat Lett 4: 175-184. Link: https://bit.ly/3usL54U

47. McDevitt M, Chattopadhyay D, Kappel B, Jaggi J, Schiffman S, et al. (2007) Tumor targeting with antibody-functionalized, radiolabeled carbon nanotubes. J Nucl Med 48: 1180-1190. Link: https://bit.ly/3kiUJIM

48. Reilly RM (2007) Carbon nanotubes: Potential benefits and risks of nanotechnology in nuclear medicine. J Nucl Med 48: 1039-1042. Link: https://bit.ly/3sr7762

49. Verma O, Kumar R, Kumar A, Chand S (2012) Assisted reproductive techniques in farm animal: From artificial insemination to nanobiotechnology. Vet World 5: 301-310. Link: https://bit.ly/3khPPWc

50. Monerris M, Arévalo F, Fernández H, Zon M, Molina $P$ (2012) Integrated electrochemical immunosensor with gold nanoparticles for the determination of progesterone. Sens Actuators B Chem 166: 586-592. Link: https://bit.ly/3uqdqIZ

51. Wang T, Zhao G, Liang X, Xu Y, Li Y, et al. (2014) Numerical simulation of the effect of superparamagnetic nanoparticles on microwave rewarming of cryopreserved tissues. Cryobiology 68: 234-243. Link: https://bit.ly/3aQIgD4

52. Weibel M, Badano J, Rintoul I (2014) Technological evolution of hormone delivery systems for estrous synchronization in cattle. Int J Livest Res 4: 2040. Link: https://bit.ly/3bxp3Fv

53. O'Connell MJ, Bachilo SM, Huffaman CB, Moore VC, Strano MS (2002) Band gap fluorescence from individual single-walled carbon nanotubes. Sci 297 593-596. Link: https://bit.ly/3aL7VNf

54. Patil S, Kore K, Kumar P (2009) Nanotechnology and its applications in veterinary and animal science. Vet World 2: 475-477. Link: https://bit.ly/20W01Gz

55. Eijkel TCJ, Berg DVA (2005) Nanofluidics: what is it and what can we expect from it? Microfluid. Nanofluid 1: 249-267. Link: https://bit.ly/37GixuM

56. Suh R, Phadke N, Ohl D, Takayama S, Smith G (2006) In vitro fertilization within microchannels requires lower total numbers and lower concentrations of spermatozoa. Hum Reprod 21: 477-483. Link: https://bit.ly/3kkaqcx

57. Schuster T, Cho B, Keller L, Takayama S, Smith G (2003) Isolation of motile sperm from semen samples using microfluidics. Reprod Biomed 7: 75-81. Link: https://bit.ly/3qON7tO

58. Jain KK (2005) Nanotechnology in clinical laboratory diagnostics. Clin Chim Acta 358: 37-54. Link: https://bit.ly/3aMxWM6

59. Lucignani G, Bombardieri E (2004) Molecular imaging: Seeing the invisible beyond the "hot spot". Q J Nucl Med Mol Imaging 48: 1-3. Link: https://bit.ly/3uvhGH4

60. Galldiks N, Stoffels G, Ruge M, Rapp M, Sabel M, et al. (2013) Role of O-(2 18F-fluoroethyl)-L-tyrosine PET as a diagnostic tool for detection of malignant progression in patients with low-grade glioma. J Nucl Med 54: 2046-2054. Link: https://bit.ly/3uvw95V

61. LeBlanc AK, Jakoby BW, Townsend DW, Daniel GB (2009) 18FDG-PET imaging in canine lymphoma and cutaneous mast cell tumor. Vet Radiol Ultrasound 50 215-223. Link: http://bit.ly/37GVJv0

62. Choi Y, Kwak J, Won J (2010) "Nanotechnology for early cancer detection." Sensors 10: 428-455. Link: http://bit.ly/3aMEcDK

63. Nie S, Xing Y, Kim G, Simons J (2007) Nanotechnology applications in cancer Annu Rev Biomed Eng 9: 257-288. Link: http://bit.ly/3bFlxbc

64. West JL, Halas NJ (2003) Engineered nanomaterials for biophotonics applications: Improving sensing, imaging, and therapeutics. Annu Rev Biomed Eng 5: 285-292. Link: http://bit.ly/3pQlq2H

65. Dianzani C, Zara G, Maina G, Pettazzoni P, Pizzimenti S, et al. (2014) Drug delivery nanoparticles in skin cancers. BioMed Res Int 2014: 895986. Link: http://bit.ly/3dLEn3z

\section{Discover a bigger Impact and Visibility of your article publication with} Peertechz Publications

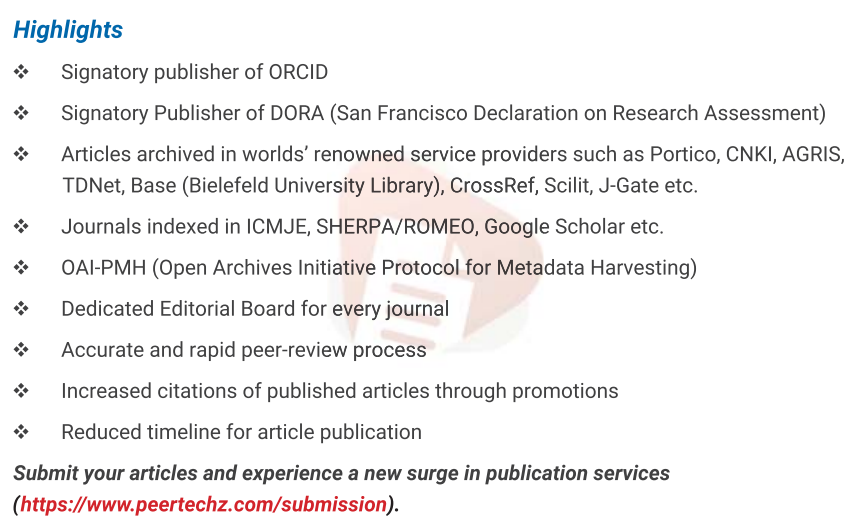

Peertechz journals wishes everlasting success in your every endeavours.

Copyright: (C) 2021 Woldeamanuel KM, et al. This is an open-access article distributed under the terms of the Creative Commons Attribution License, which permits unrestricted use, distribution, and reproduction in any medium, provided the original author and source are credited.

Citation: Woldeamanuel KM, Kurra FA, Roba YT (2021) A review on nanotechnology and its application in modern veterinary science. Int J Nanomater Nanotechnol Nanomed 7(1): 026-031. DOI: https://dx.doi.org/10.17352/2455-3492.000041 